\title{
Oral biofilms: molecular analysis, challenges, and future prospects in dental diagnostics
}

This article was published in the following Dove Press journal:

Clinical, Cosmetic and Investigational Dentistry

27 February 2013

Number of times this article has been viewed

\author{
Thuy Do' \\ Deirdre Devine' \\ Philip D Marsh ${ }^{1,2}$ \\ 'Department of Oral Biology, \\ Leeds Dental Institute, Leeds, \\ ${ }^{2} \mathrm{Health}$ Protection Agency \\ Microbiology Services, \\ Salisbury, UK
}

Correspondence: Thuy Do

Department of Oral Biology,

Leeds Dental Institute, Faculty

of Medicine and Health, University

of Leeds, Level 6 Worsley Building,

Clarendon Way, Leeds, LS2 9LU, UK

Tel +44 II 33438936

Fax +44 I| 33436548

Email n.t.do@leeds.ac.uk

\begin{abstract}
Oral biofilms are functionally and structurally organized polymicrobial communities that are embedded in an extracellular matrix of exopolymers on mucosal and dental surfaces. These biofilms are found naturally in health, and provide benefits to the host. However, this relationship can break down, and disease can occur; disease is associated with a shift in the balance of the species within these biofilms. Simple diagnostic tests have been developed that involve the culture of selected bacteria, eg, those implicated in dental caries, facilitating an assessment of risk of further disease in individual patients. However, oral diseases have a complex etiology, and because only around $50 \%$ of oral biofilm can be grown at present, culture-independent molecular-based approaches are being developed that give a more comprehensive assessment of the presence of a range of putative pathogens in samples. The diversity of these biofilms creates challenges in the interpretation of findings, and future work is investigating the ability of novel techniques to detect biological activity and function in oral biofilms, rather than simply providing a catalogue of microbial names.
\end{abstract}

Keywords: oral biofilms, dental plaque, dental diagnostics, molecular techniques, polymerase chain reaction, next-generation sequencing

\section{Introduction}

The prevalence of oral diseases has a considerable economical impact on health care provision across the world; for example, dental caries affects over $80 \%$ of the population in many countries, ${ }^{1}$ so has a significant impact on quality of life, as well as implications for systemic health. ${ }^{2}$ Indeed, chronic oral infections have been associated with diabetes and cardiovascular disease, ${ }^{3,4}$ as well as stomach ulcers and gastric cancer. ${ }^{5}$

The microbial etiology of dental diseases has been extensively studied; however, the complex nature and diverse composition of oral biofilms makes it difficult to identify the causative micro-organisms. Despite early theories focusing on identifying a single pathogen responsible for oral diseases such as dental caries, gingivitis, and chronic periodontitis, it is now generally accepted that these diseases result from the concerted actions of multispecies microbial communities. Conventional culture methods have traditionally been used to characterize the oral microbiota; however, a large proportion remains unculturable, leading to an incomplete understanding of natural microbial communities. ${ }^{6}$ Also, even when isolation of culturable organisms is achievable, one must be aware that a single species might behave differently under laboratory conditions than when it is in its natural habitat. ${ }^{7}$ Nonetheless, oral biofilms have long been the best characterized (and accessible) complex biofilms of relevance to human health and disease, but despite this, there still seems to be no effective approach to prevent oral diseases. ${ }^{8}$ The study of 
microbial communities within their own natural environment is essential to improve our knowledge of the disease process. Microbial ecology holds great potential for the development of new approaches to control oral biofilms, and for identifying novel preventive and therapeutic strategies. The enhancement of traditional microbial culture methods with the use of molecular tools has allowed a more thorough characterization of the human microbiome in general, especially that of the gut, ${ }^{9,10}$ but also of the oral cavity, ${ }^{11}$ even though it has been mostly focused on assessment of the diversity rather than the functionality of bacterial communities.

In this review, we report on the complex structure and development of oral biofilms in health and disease, and describe the molecular tools that are currently available for their analysis. We also discuss the advantages and limitations as well as the impact of such techniques on the future of patient care.

\section{Background}

The mouth, like other habitats in the body, is colonized by a characteristic and complex microbiota that grows as diverse biofilms on all mucosal and dental surfaces. This microbiota comprises protozoa, yeasts, mycoplasmas, Archaea, and bacteria, with the latter being the most numerous and diverse group. Only about half of these bacteria are currently culturable, and the application of culture-independent approaches has led to the detection of at least 1200 taxa, ${ }^{11}$ although an individual mouth harbors only a subset of these, and typically around 100 species.

The composition of microbiota varies on different surfaces due to the prevailing physical and biological conditions at distinct sites. The microbial load at mucosal sites is relatively low due to desquamation, although biofilms with a higher microbial density and diversity are found on the highly papillated surface of the tongue. In contrast, because they are nonshedding surfaces, teeth permit accumulation of the greatest amount of biomass in the mouth; the highest numbers and the greatest diversity of micro-organisms are found at stagnant sites which afford protection from oral removal forces. Fissures on occlusal surfaces have high numbers of aerobic and facultatively anaerobic, saccharolytic Gram positive bacteria, especially streptococci, and there are few anaerobes or Gram negative organisms. In contrast, the gingival crevice has the highest proportions of proteolytic and obligately anaerobic bacteria, many of which are Gram negative and some of which are unculturable. Saliva contains up to $10^{8}$ micro-organisms per $\mathrm{mL}$ that are derived from other oral surfaces, especially the tongue. ${ }^{12}$
Once established, the composition of microbial communities at a site remains relatively constant over time, and this natural balance is termed "microbial homeostasis". In general, the resident oral microbiota coexists in a harmonious relationship with the host, and makes important contributions to the normal development and general health of the host. It supports the innate and adaptive host defenses in excluding exogenous (and often pathogenic) micro-organisms, and is responsible for the natural development of the physiology of the host. However, on occasions, this harmonious relationship can breakdown, and disease can occur. In caries, there are increases in acidogenic and acid-tolerating species such as mutans streptococci, lactobacilli, and bifidobacteria, while in periodontal disease, the subgingival biofilm has elevated levels of proteolytic bacteria that subvert the host inflammatory response. ${ }^{12}$ The predominant bacteria at inflamed sites include obligately anaerobic and often Gram negative bacteria, including, for example, representatives of the genera Prevotella, Porphyromonas, Fusobacterium, Treponema, and Tannerella, and there is also an increased proportion of unculturable taxa.

\section{Development of dental biofilms}

Micro-organisms have to attach to oral surfaces if they are to persist in the mouth. The oral microbiota grows as interactive microbial communities on mucosal and dental surfaces in the form of structurally and functionally organized biofilms. The development of biofilms has been reviewed recently. ${ }^{13-16}$ All surfaces of the mouth are covered by a layer of adsorbed molecules of bacterial and salivary origin (termed the acquired pellicle), and the initial colonizing bacteria attach to this layer. Initially, these "pioneer" species are held reversibly through weak, long-range physicochemical interactions between charged molecules on the cell and oral surfaces. This interaction can become permanent via strong, shortrange stereochemical interactions between adhesins on the bacterium and complementary receptors in the acquired pellicle. These early colonizers are generally streptococci, and as they grow, they modify the local environment and make conditions suitable for colonization by more fastidious organisms. Secondary colonizers attach to receptors on these already attached bacteria (coadhesion), and gradually the diversity of the biofilm increases over time to form a multispecies community. The attached bacteria synthesize a range of extracellular polymers to form a biofilm matrix; this matrix is more than a structural scaffold because it can retain and bind many molecules, including enzymes, and so is biologically active. ${ }^{17}$ The bacteria interact, both 
synergistically and antagonistically. Bacteria combine metabolic forces in order to breakdown complex host macromolecules (eg, mucins) in order to obtain nutrients. Food chains develop, where the metabolic product of one organism becomes a primary nutrient for another. Cell-cell signaling occurs using a range of diffusible molecules which facilitates the coordination of gene expression among members of the microbial community. Peptides are used for communication between Gram positive bacteria, while autoinducer 2 is used by many Gram negative species. Thus, these oral biofilms become structurally and functionally organized, ${ }^{16}$ and their biological properties are greater than the sum of the individual species. ${ }^{14}$ These biofilms are also more tolerant of antimicrobial agents and host defenses.

\section{Advances in diagnostic techniques: molecular analysis Conventional approaches}

The invention of microscopy and the first use of appropriate selective culture media led to the improved isolation and identification of organisms found in oral biofilms. Early studies carried out extensive investigations of the composition of the microbiota in order to determine the etiological association between specific organisms and oral infection. These studies revealed an initial picture of the predominant culturable species in oral biofilms. ${ }^{18,19}$ Dental caries was associated with increases in acid-producing and acid-tolerating species, especially mutans streptococci and lactobacilli, while sites with periodontal disease had biofilms with a greater biomass, and higher numbers of proteolytic and obligately anaerobic bacteria, many of which were Gram negative. Some simple cultural "diagnostic" tests were developed using selective media for cariogenic bacteria. These tests could be set up in the dental surgery, but required a certain amount of equipment (eg, incubators) and needed time for the organisms to grow to form visible colonies, and so the results were not available while the patient was present. Generally, further tests to confirm the identification of these bacteria were not carried out, and so it was assumed that the presence of colonies on these selective media was indicative of the presence of these putative cariogenic pathogens. Patients with high salivary levels of such potentially cariogenic bacteria were designated as being at "high risk" for future caries, and were selected for additional clinical and therapeutic attention. Various chairside testing kits are available and enable rapid detection of such cariogenic bacteria. ${ }^{20-22}$

The culture of periodontal pathogens requires much more sophisticated facilities (such as those for anaerobic microbiology), and is usually carried out at specialist centers. These bacteria can take up to 10-14 days to grow, but can then be subjected to antibiotic sensitivity tests if treatment is required. In periodontal disease, the precise identification of certain organisms could be critical; for example, a particular clone (JP2) of Aggregatibacter actinomycetemcomitans is a major risk factor for localized aggressive periodontitis in young adults, ${ }^{23}$ but such identification is not easy.

\section{Contemporary approaches}

As stated earlier, not all of the bacteria in dental biofilms can be grown, especially those found in advanced caries or subgingivally, while some species are slow-growing or require specialist laboratory facilities for their cultivation. However, advances in molecular techniques over the past few decades have revolutionized our ability to detect and identify all micro-organisms in oral biofilms rapidly, including those that cannot be cultivated at present.

An example of such a technique was the use of polymerase chain reaction (PCR)-based methods in single or multiplexed approaches, which involved amplification and sequencing of fragments of the 16S rRNA gene or a housekeeping gene to help detect bacterial species associated with periodontal disease or caries, eg, members of the red complex (Porphyromonas gingivalis, Tannerella forsythia, and Treponema denticola). ${ }^{24-26}$ Diagnostic tests such as the MicroDent ${ }^{\circledR}$ Test and ParoCheck ${ }^{\circledR}$ kits using this multiplex PCR scheme are commercially available from Hain Lifescience GmbH (Nehren, Germany) and Greine Bio-one (Stonehouse, UK), respectively, and have been used to compare the microbiota between subgingival plaque samples. ${ }^{24,27,28}$ Several other molecular diagnostic tests exist and are provided by some clinicians or private companies, eg, MyPerioPath test $^{\circledR}$ and oralDNA ${ }^{\circledR}$ (OralDNA Labs, Brentwood, TN, USA) and the Integrated Periodontal ${ }^{\circledR}$ test (Biomolecular Diagnostic, Florence, Italy). ${ }^{29-31}$ They all share a common goal, which is to support the clinician in the diagnosis of oral diseases, in particular periodontal disease. The aim of these tests is to provide crucial information for advanced treatment plans and therapy for "at risk" patients, and should be used routinely as a prevention strategy for healthy patients. However, a major issue remains with their cost, which prevents them from being used widely on a routine basis. The molecular techniques used in these tests are limited to the detection of a selected number of pathogens so other important disease factors could be missed, because it is now accepted that the disease process is highly complex, and pathology arises from the actions of 
the bacterial communities and from their deregulation of the local host response. ${ }^{32}$

Much research is currently underway to further investigate oral biofilms using culture-independent approaches, and to understand microbial diversity and function as well as etiology of disease better. This effort has given rise to the development of exciting new molecular methods, which are unfortunately currently not available as diagnostic tools, because they require laboratory facilities and specialist skills, so would be impractical in the clinical setting. However, they are essential for future development and improvement of diagnostic tests. Examples of these molecular techniques include application of DNA probes specific to oral bacterial species in checkerboard DNA-DNA hybridization, which initially allowed the detection and enumeration of bacterial species from up to 43 oral biofilm samples simultaneously. ${ }^{33}$ The key advantage of this method was the ability to process an increased number of samples without the need for culture; however, the technique relied on a limited panel of preselected target species which were previously identified by cultural studies. Another drawback was the low specificity in hybridization that results from using whole genomic DNA probes on genetically diverse bacterial species. Nonetheless, this technique was widely applied by Socransky and Haffajee to explain the complex nature of the oral microbiota. ${ }^{34}$ A study by Ximénez-Fyvie et al using this technique detected all the 40 taxa in supragingival and subgingival plaque samples, and found that Actinomyces species were the most established at both oral sites, and also suggested a role of supragingival plaque as a reservoir of periodontal pathogens, with a potential to spread and infect subgingival sites. ${ }^{35}$

The beginning of the era of culture-independent techniques truly started with the implementation of 16S rRNAPCR-cloning-sequencing methodology. Because it was clear from early culture studies that not all bacterial species are able to grow in culture medium, the ability to sample and identify microbiota as accurately as possible would have an impact on the improving our understanding of microbiota-host interactions and on obtaining a more accurate picture of the microbial diversity in oral biofilms; therefore, such approaches would also impact on the future development of diagnostic tests. The PCR-cloning-sequencing method consists of amplifying the 16S rRNA genes directly from samples, taking advantage of the highly conserved regions found in the molecule. Amplicons were then cloned and sequenced via the traditional Sanger method. Giovannoni et al initially reported use of this technique in a study of microbial marine ecosystems, which revealed enormous bacterial phylogenetic diversity between members of different independent lineages. ${ }^{36}$ Since then, numerous studies have used this technique to investigate oral microbiota profiles associated with health and disease in a culture-independent approach. ${ }^{37-40}$ These studies have revealed ever-increasing species richness and complexity of oral microbiota, with numerous new operational taxonomic the units being discovered. The combined efforts from these initial investigations led to the development of the Human Oral Microbiome Database, which aims to catalogue all bacterial species found in the oral cavity. ${ }^{41}$ The major work consists of collecting 16S rRNA gene sequences into a database, to make them accessible via the Human Oral Microbiome Database website. ${ }^{11,41}$ Such an initiative is relevant for dental diagnostics because it creates a complete database of the oral microbiota which can then be used as the foundation for the future development of diagnostic methods. It originated from the Human Microbiome Project, which emphasized the necessity to identify the predominant micro-organisms in the human microbiome and to determine their relative abundance, in order to grasp the full extent of genetic and functional potential in human health and disease. Therefore, tools and methodologies must be made available to carry out the work and to obtain significant and useful clinical research data. ${ }^{11,39,42}$

More recently, a similar database was set up, known as CORE, a phylogenetically curated $16 \mathrm{~S}$ rDNA database of the core oral microbiome, ${ }^{43}$ which claimed to offer improved and more robust identification of human oral bacterial $16 \mathrm{~S}$ rRNA gene sequences compared with other methods. Its main goal is to provide a comprehensive and minimally redundant collection of oral bacteria at the genus and species level, as well as providing support for inferring community divergence and analysis of large datasets.

There are strengths and limitations associated with the 16S rRNA PCR-based technique. Some of its strong points include the ability to design specific primers for chosen groups of strains, together with its culture-independent approach when used in the direct amplification, cloning, and Sanger sequencing method, which has considerably refined our understanding of bacterial phylogenetic diversity in oral biofilms. An accurate definition of the oral microbiota in health and disease is essential for the development of dental diagnostics. The weaknesses in this method include the inability of $16 \mathrm{~S}$ rRNA gene sequencing to distinguish between closely related and highly recombinant species such as Neisseria and certain streptococci. ${ }^{44,45}$ This can be improved by use of multilocus sequence typing; ${ }^{46}$ however, there are also issues related to the definition of a species. 
Indeed, there have been questions raised concerning the validity of using species-level identification, ${ }^{47}$ because it has been recognized that only a limited number of genes might be common between members of bacterial species, ${ }^{48}$ and the remaining genes may be important in the disease process and thus be excluded from analyses using this technique. Other issues with the $16 \mathrm{~S}$ rRNA technique involve the difficulty of extracting DNA from all species and the fact that some bacterial species contain more than one copy of the 16S rRNA gene, which should perhaps be taken into consideration and further investigated when carrying out phylogenetic analyses, because they may be wrongly assumed to be identical. Overall, the technique is costly, and the cloning stage is also hugely tedious; further, it is limited to low sample numbers and only reveals the predominant organisms in a sample. Despite these issues, sequencing data have been made publicly available and have helped in the identification of most members of the oral microbial community. Through the design of probes for detecting hundreds of bacterial species, it has helped to expand the range of the checkerboard assay into a microarray format, ie, the Human Oral Microbe Identification Microarray, ${ }^{49}$ which uses reverse-capture ribosomal RNA probes to identify and evaluate the relative abundance of 272 oral taxa. Several studies have used the Human Oral Microbe Identification Microarray in their investigation of the microbial etiology of oral diseases. Colombo et al observed a distinct microbial profile between samples from refractory periodontitis, severe periodontitis, and healthy patients. ${ }^{49}$ The microarray method has also been used in other studies for oral bacterial profiling, ${ }^{50,51}$ and in in vitro multispecies microcosm models. ${ }^{52}$

\section{Next-generation sequencing methods}

Recent advances in technology have paved the way for new possibilities in microbiome research through the development of high-throughput sequencing or next-generation sequencing. The pyrosequencing methods (as provided by the 454 system [Roche, Basel, Switzerland], the first and most widely used next-generation sequencing platform) are based on the detection of pyrophosphate released during DNA synthesis. First, DNA libraries are constructed from the sample, the DNA fragments are then amplified within an emulsion, and sequencing by synthesis occurs in a massively parallel manner, where visible light is produced and detected, generating gigabytes of nucleotide sequence reads data. ${ }^{53}$ The data output is then processed through a bioinformatics pipeline, which consists of quality screening to remove low-quality reads. The remaining data are separated into clusters, and assigned into operational taxonomic units after comparison with sequences from available databases, such as the Human Oral Microbiome Database or the Ribosomal Database Project. ${ }^{54}$

Initial settings of this technique only produced short sequence reads of about 150 base pairs, and hence could only enable targeting of a single variable region of the 16S rRNA gene for taxonomic profiling studies. For example, Keijser et al reported an overly diverse microbiota in healthy adults than previously recorded (19,000 phyla detected), using the V6 region of the $16 \mathrm{~S}$ rRNA gene. ${ }^{55}$ More recently, with the increase in read length because of improvements in technology, larger regions of the gene can be sequenced. Some studies have carried out bacterial community profiling using sequences from the hypervariable V5-V6 region, hence increasing the phylogenetic resolution. ${ }^{56,57}$ Saber et al observed the microbiota of symptomatic periapical lesions, and found that the predominant organisms included anaerobic bacteria, with high proportions of streptococci, actinomyces, and unknown taxa. ${ }^{58} \mathrm{~A}$ comparison study between pyrosequencing and the Human Oral Microbe Identification Microarray was carried out by Ahn et al, determining the oral microbiome community profiles of patients with oral cancer and in healthy volunteers using both techniques. ${ }^{59}$ The major phyla (Firmicutes, Proteobacteria, Bacteroidetes, Actinobacteria, and Fusobacteria) were detected by the two methods; however, pyrosequencing provided superior sensitivity in the level of identification.

Other studies have used the Illumina platform to perform taxonomic profiling by $16 \mathrm{~S}$ rRNA amplicon sequencing, ${ }^{60,61}$ using different hypervariable regions. This next-generation sequencing platform uses a different chemistry involving a reversible terminator-based method during sequencing by synthesis, resulting in shorter read lengths compared with the 454 pyrosequencing platform, but yields a much higher data output. Recently, the available HiSeq 2500 provided by Illumina offers a maximum read length of $2 \times 150$ base pairs, and a data output of up to 600 gigabytes. ${ }^{62}$

Despite claims of reliability, lower costs, ease of use, and shorter time frame of protocols, ${ }^{63}$ these high-throughput techniques require a high level of expertise, and cannot be transferred to a clinical setting, especially when complex bioinformatic skills and computer power are required to make sense of the deluge of data generated. A great advantage of these techniques lies in the fact that they are able to deal with an increasing number of samples, lowering the cost with the use of a multiplexed barcoded sequencing approach, allowing numerous samples to be pooled together into a single run. 
The major limitations remain the short reads produced, and the errors in reads sequences which are caused by formation of amplification artifacts and chimeric sequences from PCR with mixed templates. Software and algorithms such as PyroNoise ${ }^{64}$ and deNoiser ${ }^{65}$ are now available to correct these faults and aim to provide a more accurate survey of the composition of the oral microbiota. However, in spite of performing rigorous quality control steps on the data (applying the appropriate parameters from bioinformatics correction tools), this technique is not perfect and cannot avoid huge overestimation of the real microbial diversity, because of sequencing errors. Also, the discriminatory power of a single gene or gene fragment, as described earlier, is not always sufficient to differentiate accurately between some closely related species.

Nevertheless, milestones have been achieved in our understanding of the structure and composition of complex oral biofilms. Further and deeper knowledge of the metabolic activities regulating and governing biological processes at the species-species level are now needed in order to uncover the mechanism of transition from health to disease, and their interaction with the responses of the host. So far, all the effort has been focused on identifying members of the oral microbiome, and making etiological associations between various organisms and oral diseases.

Rather than just determining the taxa present in biofilms, it may be more useful to understand the functions and activities at work. Recent publications have reported such attempts, using a metagenomic approach, ie, applying nextgeneration sequencing to sequence all the microbial DNA within a sample directly, and reconstructing the genomes of the microbiota via de novo assembly or mapping against a reference genome database. The resulting data can be processed further into functional gene annotation, and this method is claimed to offer an insight into the global genetic, metabolic, and ecological modifications occurring within subgingival plaque samples in periodontitis. ${ }^{66}$

An increase in virulence factors in the disease microbiome was also observed, perhaps resulting from or contributing to the disruption of microbial homeostasis. Belda-Ferre et al identified etiological agents of caries and confirmed the view that caries is a polymicrobial disease. ${ }^{8}$ Their oral metagenomic data revealed distinct functional categories when compared with the gut microbiome. They also found that Streptococcus mutans, a bacterial species widely implicated in the initiation and development of caries, was not the predominant species in caries, suggesting that in order to understand disease it might be more important to decipher microbial function in oral biofilms rather than merely listing the taxa that are present. They also observed that the microbiome of healthy individuals with no prior history of caries showed an overrepresentation of genes involved in quorum sensing and coding for antimicrobial peptides.

Luo et al used the 454 and Illumina platforms on the same microbial community sample for metagenomic analysis to assess and compare their performance. ${ }^{67}$ Both these high-throughput techniques generated consistent data, but Illumina provided longer and more accurate contigs, despite yielding shorter read lengths than the 454 platform. As well as being more cost-effective, the data obtained from Illumina might be more appropriate for carrying out metagenomic studies, in particular without the bias associated with PCR-based methods and cloning. Nonetheless, there are issues associated with direct metagenome sequencing because of the sampling protocols used, which cannot guarantee inclusion of all microbial species, as well as the technique itself, which only yields partial genome sequences, inevitably leading to inconsistent annotations. ${ }^{68}$ Moreover, it may be incorrect to assume that the functions assigned to the sequenced metagenome are exerted within the studied microbiome, without confirmation that the genes are actually expressed under the given environmental conditions and time frame. In order to understand such complex interactions fully, and capture the subtleties of biochemical activities occurring within oral biofilms in health and disease, more developments are needed in term of tools and technologies. The goal will be to use the ever-advancing techniques available to decipher the whole meta-transcriptome, meta-proteome, and meta-metabolome. The view of microbial communities as one meta-organism is appropriate, with all the meta-omic disciplines becoming the ultimate target of investigation for understanding the cellular processes at work within the biofilm and to link the data generated with prediction of functional responses to environmental stress. Such developments may conceivably accelerate the development of diagnostic tests that rely on detection of a small number of disease-specific functions in the microbial community, and do not require information regarding species composition. However, the major hurdle lies in the difficulty in analysis and interpretation of the enormous amount of data generated.

Investigations of microbial metatranscriptomes are not novel, and numerous studies have applied the RNASeq method to the gut microbiota. ${ }^{69-71}$ The technique consists of extracting mRNA fragments from clinical samples and reverse-transcribing them into cDNA which 
is then sequenced in a high-throughput and massively parallel manner. However, this has not been applied to the oral microbiome yet.

Recently, an impressive new cutting-edge procedure was described by Sherstnev et al, implying direct sequencing of RNA extracted from the plant Arabidopsis thaliana, thus bypassing the reverse-transcription step. ${ }^{72}$ The emerging field of single-cell genomics is also currently being implemented for bacteria and Archaea. ${ }^{73}$ It may not be long before these exciting techniques are also used for further characterization of oral biofilms but, until then, diagnostic tests based on identification of specific organisms using culture and basic molecular methods remain the norm. The issue with these commercially available tests is the question of their true value in terms of reliability for detecting agents causative of disease, given our limited knowledge of the complex ecosystem involved. The other major concern lies in the ability of the clinician or diagnostic company to interpret the results correctly and in such a way as to provide benefit for patients.

\section{Implications for enhanced patient care}

Molecular techniques in microbiology have many advantages over traditional culture methods. Indeed, they provide more accurate results, and with the recent advances in technology, have the potential to lead the way to development of more personalized treatments through a better understanding of genetics, biochemistry, pathogenicity, phylogeny, and microbial population structure and evolution. However, only a limited number of commercial diagnostic aids are available, and new improved kits that give an overall indication of disease status (including metabolic pathways and activities) are needed. Despite the huge improvement in identification of the composition of oral biofilms, culture methods will still play an essential role in every research laboratory and in the clinical setting, and be part of the initial background baseline diagnostic screening and analysis steps in assisting patient therapy.

As described in this review, molecular methods for investigating oral biofilms are not perfect, and come with many drawbacks, including cost, their time-consuming nature, and the high level of expertise required to process samples and carry out data analysis. Also, their impracticality and our current inability to use these techniques in a clinical setting will impact on the enthusiasm of dentists to implement such diagnostic tools, and the benefit to patients from the use of such tests still needs to be quantified.

A major obstacle to developing a simple chairside test is the huge complexity in procedures implicated in the development of diagnostics, which can be time-consuming and costly. It is essential that more effort is directed towards identifying biomarkers of disease and their role in diagnosis, in order to help improve the outcome of patient therapy, and to establish a relationship between the mechanism of disease progression and therapeutic intervention.

Rather than improving the diagnosis of conventional oral diseases, the future of diagnostics in dentistry may be related to more holistic medical issues. The ease of sampling oral fluids such as saliva and gingival crevicular fluid makes these the preferred biological samples for assessing biomarkers. ${ }^{74}$ Oral biofilms are also easy for dental professionals to sample, and if chairside tests, such as functional property assays, were available to enable rapid analysis of biofilm samples, this would help guide treatment and therapy. Other important factors are the need for further prospective cohort studies and standardization of protocols for sample collection and handling, in order to obtain a better comparison between future studies. Indeed, improved patient screening and appropriate clinical samples would certainly allow a better understanding of health and disease. This will ultimately lead to novel therapies and guidance on lifestyle changes, implicating the microbiome.

Next-generation sequencing technologies have current applications in medical diagnostics, eg, in genetic diseases, but they are not yet applied to dentistry. However, with time, these advanced technologies will become faster, more accurate, easier to use, and more affordable, which will allow clinicians to provide patients with rapid personalized treatment and prevention plans. Moreover, the data generated by these advanced molecular techniques may also contain human gene information, which together with microbial genetic and functional information can provide a more complete understanding of our predisposition to oral diseases. There are many public health challenges yet to overcome. As mentioned in this review, the main difficulties include the polymicrobial nature of oral diseases, and so their nonspecific etiology, the potentially high cost of tests, and the specialist skills required for data interpretation. However, the public health benefits associated with future prospects in dental diagnostics for oral biofilms would include the more rational selection of treatment plans through a systematic differentiation of "active" from "inactive" lesions and identification of "at risk" sites. Other benefits would see the underpinning of the principles for "minimally invasive dentistry", with the potential for reducing overtreatment, and ultimately resulting in cheaper treatments over the life of a patient through evidence-based dentistry. 
Despite the great challenges ahead, the combined efforts and collaboration of clinicians and researchers will be essential in providing a platform for creating future oral diagnostics and find applications for moving from bench to clinical practices. It is likely that clinicians will have to rely on external companies which will be able to accommodate rapid sample processing and analyses. Current diagnostic tools only target specific organisms, but future methods will probably depend on a more radical shift in disease concepts, and focus on detection of microbial functions in oral biofilms rather than microbial names.

\section{Disclosure}

The authors report no conflicts of interest in this work.

\section{References}

1. Petersen P, Lennon M. Effective use of fluorides for the prevention of dental caries in the 21st century: The WHO approach. Community Dent Oral Epidemiol. 2004;32(5):19-21.

2. Flemmig T, Beikler T. Control of oral biofilms. Periodontol 2000. 2011; 55(1):9-15.

3. Nakano K, Nomura R, Matsumoto M, Ooshima T. Roles of oral bacteria in cardiovascular diseases - from molecular mechanisms to clinical cases: Cell-surface structures of novel serotype k Streptococcus mutans strains and their correlation to virulence. J Pharmacol Sci.2010; 113(2):120-125.

4. Li X, Kolltveit K, Tronstad L, Olsen I. Systemic diseases caused by oral infection. Clin Microbiol Rev. 2000;13(4):547-558.

5. Watabe K, Nishi M, Miyake H, Hirata K. Lifestyle and gastric cancer: a case-control study. Oncol Rep. 1998;5(5):1191-1194.

6. Hiyari S, Bennett K. Dental diagnostics: molecular analysis of oral biofilms. J Dent Hyg. 2011;85(4):256-263.

7. Siggins A, Gunnigle E, Abram F. Exploring mixed microbial community functioning: recent advances in metaproteomics. FEMS Microbiol Ecol. 2012;80(2):265-280.

8. Belda-Ferre P, Alcaraz L, Cabrera-Rubio R, et al. The oral metagenome in health and disease. ISME J. 2012;6(1):46-56.

9. Lepage P, Leclerc M, Joossens M, et al. A metagenomic insight into our gut's microbiome. Gut. 2013;62(1):146-158.

10. Dave M, Higgins P, Middha S, Rioux K. The human gut microbiome: current knowledge, challenges, and future directions. Transl Res. 2012;160(4):246-257.

11. Dewhirst F, Chen T, Izard J, et al. The human oral microbiome. J Bacteriol. 2010;192(19):5002-5017.

12. Marsh P, Martin M. Oral Microbiology, 5th ed. Oxford, UK: Churchill Livingstone; 2009.

13. Kolenbrander P, Palmer R, Periasamy S, Jakubovics N. Oral multispecies biofilm development and the key role of cell-cell distance. Nat Rev Microbiol. 2010;8:471-480.

14. Marsh P. Dental plaque: biological significance of a biofilm and a community life-style. J Clin Periodontol. 2005;32(6):7-15.

15. Marsh P, Moter A, Devine D. Dental plaque biofilms - communities, conflict and control. Periodontol 2000. 2011;55(1):16-35.

16. Zijnge V, van Leeuwen $M$, Degener J, et al. Oral biofilm architecture on natural teeth. PLoS One. 2010;5:e9321.

17. Flemming H, Wingender J. The biofilm matrix. Nat Rev Microbiol. 2010;8:623-633.

18. Brecx M, van Oosten M. Microbiological findings of subgingival plaque during long-term experimental gingivitis in humans. Oralprophylaxe. 1989;11(4):127-130. German.

19. Moore W, Holdeman L, Cato E, Smibert R, Burmeister J, Ranney R. Bacteriology of moderate (chronic) periodontitis in mature adult humans. Infect Immun. 1983;42(2):510-515.
20. Walsh L, Tsang A. Chairside testing for cariogenic bacteria: Current concepts and clinical strategies. J Minim Interv Dent. 2008;1(2):126-149.

21. Matsumoto Y, Sugihara N, Koseki M, Maki Y. A rapid and quantitative detection system for Streptococcus mutans in saliva using monoclonal antibodies. Caries Res. 2006;40(1):15-19.

22. Shi W, Jewett A, Hume W. Rapid and quantitative detection of Streptococcus mutans with species specific monoclonal antibodies. Hybridoma. 1998;17(1):365-371.

23. Haubeck D. The highly leukotoxic JP2 clone of Aggregatibacter actinomycetemcomitans: evolutionary aspects, epidemiology and etiological role in aggressive periodontitis. APMIS Suppl. 2010;130:1-53.

24. Eick S, Pfister W. Comparison of microbial cultivation and a commercial PCR based method for detection of periodontopathogenic species in subgingival plaque samples. J Clin Periodontol. 2002;29(7): 638-644.

25. García L, Tercero J, Legido B, Ramos J, Alemany J, Sanz M. Rapid detection of Actinobacillus actinomycetemcomitans, Prevotella intermedia and Porphyromona gingivalis by multiplex PCR. J Periodontal Res. 1998;33(1):59-64.

26. Rôças I, Siqueira JJ, Santos K, Coelho A. "Red complex" (Bacteroides forsythus, Porphyromonas gingivalis, and Treponema denticola) in endodontic infections: A molecular approach. Oral Surg Oral Med Oral Pathol Oral Radiol Endod. 2001;91(4):468-471.

27. Greiner Bio-one. Parocheck diagnostic kit for the identification of 10-20 periodontal pathogens, 2012. Available from: http://www. greinerbioone.com/en/germany/articles/catalogue/articles/351_1. Accessed January 24, 2013.

28. Hain Lifescience GmbH. Periodontitis diagnostics and therapy, 2012. Available from: http://www.hain-lifescience.de. Accessed January 24, 2013.

29. Biomolecular Diagnostic. Microbiological and genetic tests [Integrated Periodontal test]. Florence, Italy: 2006. Available from: http://www. biomoleculardiagnostic.com. Accessed January 24, 2013.

30. OralDNA Labs Inc. Clinical Diagnostic Laboratory. MyPerioPath test. Brentwood, TN, 2012. Available from: http://www.OralDNA.com. Accessed January 24, 2013.

31. Sanderson J. MyPerioPath test. Salivary DNA tests to help diagnose periodontal disease. Birmingham, AL: SanDentistry; 2012. Available from: http://www.sandentistry.com. Accessed January 24, 2013.

32. Hajishengallis G, Darveau RP, Curtis MA. The keystone-pathogen hypothesis. Nat Rev Microbiol. 2012;10(10):717-725.

33. Socransky S, Smith C, Martin L, Paster B, Dewhirst F, Levin A. "Checkerboard" DNA-DNA hybridization. Biotechniques. 1994; 17(4):788-792.

34. Socransky SS, Haffajee AD. Periodontal microbial ecology. Periodontol 2000. 2005;38:135-187.

35. Ximénez-Fyvie L, Haffajee A, Socransky S. Microbial composition of supra- and subgingival plaque in subjects with adult periodontitis. J Clin Periodontol. 2000;27(10):722-732.

36. Giovannoni S, Britschgi T, Moyer C, Field K. Genetic diversity in Sargasso Sea bacterioplankton. Nature. 1990;345(6270):60-63.

37. de Lillo A, Booth V, Kyriacou L, Weightman A, Wade W. Cultureindependent identification of periodontitis-associated Porphyromonas and Tannerella populations by targeted molecular analysis. $J$ Clin Microbiol. 2004;42(12):5523-5527.

38. Riggio M, Lennon A, Rolph H, et al. Molecular identification of bacteria on the tongue dorsum of subjects with and without halitosis. Oral Dis. 2008;14(3):251-258.

39. TannerA, Mathney J, Kent R, et al. Cultivable anaerobic microbiota of severe early childhood caries. J Clin Microbiol. 2011;49(4):1464-1474.

40. Vartoukian S, Palmer R, Wade W. Diversity and morphology of members of the phylum "synergistetes" in periodontal health and disease. Appl Environ Microbiol. 2009;75(11):3777-3786.

41. Human Oral Microbiome Database. Human oral microbiome database at the Forsyth Institute (USA), 2012. Available from: http://www.homd. org. Accessed January 24, 2013. 
42. Chen T, Yu W, Izard J, Baranova O, Lakshmanan A, Dewhirst F. The Human Oral Microbiome Database: a web accessible resource for investigating oral microbe taxonomic and genomic information. Database (Oxford). 2010;2010:baq013.

43. Griffen A, Beall C, Firestone N, et al. CORE: a phylogenetically-curated $16 \mathrm{~S}$ rDNA database of the core oral microbiome. PLoS One. 2011;6(4):e19051.

44. Do T, Jolley K, Maiden M, et al. Population structure of Streptococcus oralis. Microbiology. Microbiology. 2009;155(8):2593-2602.

45. Hanage W, Fraser C, Spratt B. Fuzzy species among recombinogenic bacteria. BMC Biol. 2005;3:6.

46. Maiden M, Bygraves J, Feil E, et al. Multilocus sequence typing: a portable approach to the identification of clones within populations of pathogenic microorganisms. Proc Natl Acad Sci U S A. 1998;95(6):3140-3145.

47. Wade W. Has the use of molecular methods for the characterization of the human oral microbiome changed our understanding of the role of bacteria in the pathogenesis of periodontal disease? J Clin Periodontol. 2011;38(11):7-16.

48. Tettelin H, Masignani V, Cieslewicz M, et al. Genome analysis of multiple pathogenic isolates of Streptococcus agalactiae: implications for the microbial "pan-genome". Proc Natl Acad Sci U S A. 2005;102(39): 13950-13955.

49. Colombo A, Boches S, Cotton S, et al. Comparisons of subgingival microbial profiles of refractory periodontitis, severe periodontitis, and periodontal health using the human oral microbe identification microarray. J Periodontol. 2009;80(9):1421-1432.

50. Holgerson P, Vestman N, Claesson R, et al. Oral microbial profile discriminates breastfed from formula-fed infants. J Pediatr Gastroenterol Nutr. 2013;56(2):127-136.

51. Luo A, Yang D, Xin B, Paster B, Qin J. Microbial profiles in saliva from children with and without caries in mixed dentition. Oral Dis. 2012; 18(6):595-601.

52. Rudney J, Chen $\mathrm{R}$, Lenton $\mathrm{P}$, et al. A reproducible oral microcosm biofilm model for testing dental materials. J Appl Microbiol. 2012;113(6):1540-1553.

53. Ronaghi M. Pyrosequencing sheds light on DNA sequencing. Genome Res. 2001;11(1):3-11.

54. Cole J, Wang Q, Cardenas E, et al. The Ribosomal Database Project: improved alignments and new tools for rRNA analysis. Nucleic Acid Res. 2009;37:D141-D145.

55. Keijser B, Zaura E, Huse S, et al. Pyrosequencing analysis of the oral microflora of healthy adults. J Dent Res. 2008;87(11):1016-1020.

56. Crielaard W, Zaura E, Schuller A, Huse S, Montijn R, Keijser B. Exploring the oral microbiota of children at various developmental stages of their dentition in the relation to their oral health. BMC Med Genomics. 2011;4:22.

57. Ozok A, Persoon I, Huse S, et al. Ecology of the microbiome of the infected root canal system: a comparison between apical and coronal root segments. Int Endod J. 2012;45(6):530-541.
58. Saber M, Schwarzberg K, Alonaizan F, et al. Bacterial flora of dental periradicular lesions analyzed by the 454-pyrosequencing technology. J Endod. 2012;38(11):1484-1488.

59. Ahn J, Yang L, Paster B, et al. Oral microbiome profiles: 16S rRNA pyrosequencing and microarray assay comparison. PLoS One. 2011;6(7):e22788

60. Cockburn A, Dehlin J, Ngan T, et al. High throughput DNA sequencing to detect differences in the subgingival plaque microbiome in elderly subjects with and without dementia. Investig Genet. 2012;3(1):19.

61. Lazarevic V, Whiteson K, Huse S, et al. Metagenomic study of the oral microbiota by Illumina high-throughput sequencing. J Microbiol Methods. 2009;79(3):266-271.

62. Illumina Inc. 2012. http://www.illumina.com.

63. Heng N, Stanton J. Oral bacterial genome sequencing using the highthroughput Roche Genome Sequencer FLX System. Methods Mol Biol. 2010;666:197-218.

64. Quince C, Lanzén A, Curtis T, et al. Accurate determination of microbial diversity from 454 pyrosequencing data. Nat Methods. 2009;6(9):639-641.

65. Reeder J, Knight R. Rapidly denoising pyrosequencing amplicon reads by exploiting rank-abundance distributions. Nat Methods. 2010; 7(9):668-669.

66. Liu B, Faller L, Klitgord N, et al. Deep sequencing of the oral microbiome reveals signatures of periodontal disease. PLoS One. 2012;7(6):e37919.

67. Luo C, Tsementzi D, Kyrpides N, Read T, Konstantinidis K. Direct comparisons of Illumina vs Roche 454 sequencing technologies on the same microbial community DNA sample. PLoS One. 2012;7(2):e30087.

68. Zaura E. Next-generation sequencing approaches to understanding the oral microbiome. Adv Dent Res. 2012;24(2):81-85.

69. Bomar L, Maltz M, Colston S, Graf J. Directed culturing of microorganisms using metatranscriptomics. MBio. 2011;2(2):e00012-e00011.

70. McNulty N, Yatsunenko T, Hsiao A, et al. The impact of a consortium of fermented milk strains on the gut microbiome of gnotobiotic mice and monozygotic twins. Sci Transl Med. 2011;3(106):106ra106.

71. Rey F, Faith J, Bain J, et al. Dissecting the in vivo metabolic potential of two human gut acetogens. J Biol Chem. 2010;285(29):22082-22090.

72. Sherstnev A, Duc C, Cole C, et al. Direct sequencing of Arabidopsis thaliana RNA reveals patterns of cleavage and polyadenylation. Nat Struct Mol Biol. 2012;19(8):845-852.

73. Blainey $P$. The future is now: single-cell genomics of bacteria and archaea. FEMS Microbiol Rev. January 8, 2013. [Epub ahead of print.]

74. Thomadaki K, Bosch JA, Oppenheim F, Helmerhorst E. The diagnostic potential of salivary protease activities in periodontal health and disease Oral Diseases. January 15, 2013. [Epub ahead of print.] doi: 10.1111/ odi.12069
Clinical, Cosmetic and Investigational Dentistry

\section{Publish your work in this journal}

Clinical, Cosmetic and Investigational Dentistry is an international, peer-reviewed, open access, online journal focusing on the latest clinical and experimental research in dentistry with specific emphasis on cosmetic interventions. Innovative developments in dental materials, techniques and devices that improve outcomes and patient satisfac-

\section{Dovepress}

tion and preference will be highlighted. The manuscript management system is completely online and includes a very quick and fair peerreview system, which is all easy to use. Visit http://www.dovepress. com/testimonials.php to read real quotes from published authors. 